\author{
Margarete Flöter-Durr \\ Universität Straßburg \\ margarete.floeter-durr@etu.unistra.fr
}

\title{
DER BEGRIFF DER RELEVANZ UND DESSEN ANWENDUNG AUF DIE RECHTSÜBERSETZUNG
}

DOI: http://dx.doi.org/10.12775/RP.2017.008

\begin{abstract}
Zarys treści: Metodologicznym punktem rozważań w pracy jest pojęcie „ekwiwalencji” i krytyczne spojrzenie na nie w teorii przekładu. Różnorodność opinii na ten temat skutkuje nieprzydatnością tego określenia w teorii przekładu. Autorka proponuje spojrzeć na problem z punktu widzenia pojęcia „odpowiedniość” i jej typologii przedstawionej przez Alfreda Schütza. To zagadnienie bowiem wywołuje dyskusje na temat związku między odpowiedniością i orientacją. W końcowej części pracy poruszono sprawę mechanizmu działania odpowiedniości w tłumaczeniu prawniczym. Problem ukazano na przykładach z praktyki tłumaczeniowej w parach języków: niemiecki-francuski i francuski-niemiecki.
\end{abstract}

Słowa kluczowe: ekwiwalencja, odpowiedniość, geneza i mechanizm sensu, orientacja, przekład prawniczy, interpretacja, temat, motywacja, kreatywność

In dem vorliegenden Beitrag soll der Begriff der Relevanz nach Alfred

Schütz (2004a) vorgestellt und seine mögliche Anwendung auf die Rechtsübersetzung anhand von praktischen Beispielen aus der Übersetzungspraxis im Sprachenpaar Deutsch und Französisch veranschaulicht werden.

Bevor der Begriff der Relevanz in die Diskussion eingeführt wird, gehen wir methodologisch von dem linguistischen Paradigma der Übersetzung, insbesondere von dem äquivalenztheoretischen Ansatz, aus. Der Begriff der Äquivalenz wird einer Kritik unterzogen und die Gründe für sein Scheitern in der Übersetzungstheorie werden kurz aufgezeigt. Anschließend werden der Schützsche Begriff der Relevanz und die von ihm erarbeitete Typologie der Relevanzen sowie die Voraussetzungen einer erfolgreichen Kommunika- 
tion erläutert. Im Weiteren wird auf die Verzahnung von Relevanz und Orientierung und deren Rolle in der Rechtsübersetzung eingegangen.

\section{Der Begriff der Äquivalenz in der Übersetzungstheorie}

Der äquivalenztheoretische Ansatz ist nach Siever, neben dem texttypologischen Ansatz, eine der Ausprägungen des linguistischen Paradigmas in der Übersetzungstheorie (2010:46). Der Begriff der Äquivalenz und der Begriff der Invarianz stellen die Grundpfeiler dieses Ansatzes dar.

Traditionell wird das Übersetzen als eine „Suche nach Äquivalenten“ (Reiss 1971: 54) aufgefasst. Das bedeutet, dass sich der Übersetzungsvorgang in dem Auffinden der Äquivalente erschöpft (Siever 2010: 46). Häufig wird auch behauptet, der Äquivalenzbegriff stelle „das Kernstück aller Übersetzungstheorie“ dar (Siever 2010: 78). Dieser Begriff findet sich bereits bei Jakobson, der ihn in seinem Artikel On Linguistics Aspects of Translation (1959) folgendermaßen definiert hat: „Äquivalenz in der Differenz stellt das wichtigste Problem der Sprache und das zentrale Problem der Linguistik dar"1. Nida hat diesen Begriff aufgegriffen und sah darin eine Übersetzungstechnik, mit der eine möglichst natürliche Ausdrucksweise in der Zielsprache erreicht werden sollte. Diese Technik besteht darin „(..) einen semantisch und stilistisch gleichwertigen Ausdruck in der Zielsprache zu finden"2 (Nida 1964: 68). In Ermangelung identischer Äquivalente besteht die Aufgabe des Übersetzers darin, das bestmögliche Äquivalent zu finden (Nida 1964: 159). Um dies zu ermöglichen, differenziert Nida zwischen formaler und dynamischer Äquivalenz. Die formale Äquivalenz besteht in einer form- und inhaltsgetreuen Wiedergabe, wohingegen die dynamische Äquivalenz auf das „Prinzip der gleichwertigen Wirkung ${ }^{{ }^{3} 3}$ (1964: 159) abstellt. Übersetzungen, die mit der dynamischen Äquivalenz operieren, orientieren sich an der Reaktion des Zieltextrezipienten und stellen „das bestmögliche natürliche Äquivalent der Mitteilung in der Ausgangssprache ${ }^{“ 4}$ (Nida 1964: 166) dar, wobei der Akzent hier auf „natürlich“ liegt. Dies erscheint auch mit der Feststellung von

\footnotetext{
${ }^{1}$ Unsere Übersetzung der Originalpassage: „Equivalence in difference is the cardinal problem of language and the pivotal concern of linguistics".

${ }^{2}$ Unsere Übersetzung der Originalpassage: ,to generate the stylistically and semantically equivalent expression in the receptor language“.

${ }^{3}$ Unsere Übersetzung der Originalpassage: „principle of equivalent effect".

${ }^{4}$ Unsere Übersetzung der Originalpassage: ,the closest natural equivalent to the sourcelanguage message“.
} 
Wittgenstein im $\$ 116$ der Philosophischen Untersuchungen konsistent. Denn als Übersetzer müssen wir uns stets fragen: „Wird denn dieses Wort in der Sprache, in der es seine Heimat hat, ja tatsächlich so gebraucht?"“ (2015a:82). Demzufolge kommt dem Übersetzer nach Nida eine zentrale Rolle im Übersetzungsprozess zu (1964: 145).

Im Gegensatz zu Nida verfolgt Catford einen „streng linguistischsprachbezogenen Ansatz in Anlehnung an die sprachwissenschaftlichen Arbeiten des englischen Kontextualismus (Firth, Halliday)" (Siever 2010: 42). Nach Catford kommt der Äquivalenzbeziehung zwischen Ausgans- und Zieltext eine erhebliche Bedeutung zu, sodass das zentrale Problem der Übersetzungspraxis darin besteht, „Übersetzungsäquivalente zu finden“ (1965:21). Catford entwickelt einen systemlinguistischen Bedeutungsbegriff, „demzufolge Bedeutung nur ein einzelsprachliches Phänomen ist“" (Siever 2010: 43). Somit liege zwischen dem Ausgangs- und dem Zieltext keine Bedeutungsidentität vor, sondern es gibt zwei Bedeutungen: „Ein Ausgangstext hat eine Bedeutung in der Ausgangssprache und ein Zieltext hat eine Bedeutung in der Zielsprache “5 (1965:35). In der Konsequenz besteht die Übersetzung nicht in einem interlingualen Bedeutungstransfer, sondern darin, eine Funktionsäquivalenz herzustellen. Das bedeutet, dass „Übersetzungsäquivalente in derselben Situation funktionieren können müssen" (Siever 2010: 43). Die Funktionsäquivalenz bewirkt, dass Begriffe wie Situation und Kontext in den Mittelpunkt der Theorie von Catford gestellt werden. Auf ihn geht auch die Unterscheidung zwischen Kontext und Ko-Text zurück. Die Leistungen des Übersetzers sowohl mit Blick auf den Verstehensprozess als auch auf die Interpretation des Ausgangstextes spielen bei Catford keine Rolle bzw. „werden von ihm systematisch ignoriert" (Siever 2010: 43). Letztendlich hat der Ansatz von Catford „lediglich zu einem spröden Ebenen-Modell des Übersetzens geführt (...), in dem weder dem Übersetzungsprozess noch dem Übersetzer besondere Beachtung geschenkt wird (Siever 2010: 42). Der Begriff der Äquivalenz, insbesondere die von Nida vorgeschlagene dynamische Äquivalenz hat nichtsdestotrotz eine wichtige Wende in der Übersetzungstheorie eingeleitet. Denn damit ist sie von dem Paradigma der Wörtlichkeit bzw. der Treue abgerückt und hat den Übersetzer in den Mittelpunkt des Übersetzungsprozesses gestellt.

${ }^{5}$ Unsere Übersetzung der Originalpassage: „An SL text has an SL meaning and a TL text has a TL meaning". 


\subsection{Kritik des Äquivalenzbegriffs}

Ursprünglich entstammt der Begriff der Äquivalenz der formalen Logik, in der er eine bilaterale Implikation von der Art „wenn p, dann q“ und um-

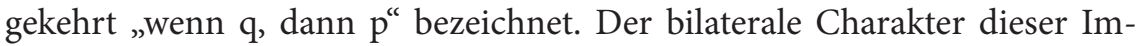
plikation verweist auf eine bidirektionale Beziehung bzw. eine umkehrbare Zuordnung von Elementen. Dieser Begriff wurde dann von der Übersetzungstheorie übernommen, wobei diese Übernahme unkritisch erfolgt ist. Obwohl es in der Sprache keine umkehrbare Zuordnung von sprachlichen Ausdrücken gibt, verwendet die Übersetzungstheorie dennoch den Begriff der Äquivalenz, um eine Identitäts-Hypothese zu beschreiben und lässt dabei die dem logischen Begriff innewohnende Bidirektionalität vollkommen unberücksichtigt. Nach Siever ist der problematische Charakter des Begriffs der Äquivalenz auf ein erkenntnistheoretisches Problem zurückzuführen. Übersetzen wird - wie bereits oben erwähnt - traditionell als das Auffinden von Äquivalenten betrachtet. Dieses Auffinden beruht auf dem grundsätzlichen Postulat, dass „Äquivalenzbeziehungen zwischen den Sprachen auf der Ebene der „langue“ (der Sprache als System) bestehen (Reiss 1971: 54). Das erkenntnistheoretische Problem resultiert aus der Annahme, dass außerhalb der Sprache „eine sprach- und interpretationsunabhängige Vergleichsentität bestehe“. Diese Vergleichsentität ist die Welt, auf die Bezug genommen wird und die der Übersetzung ontologisch vorgelagert ist. Dieses Problem stellt sich in der Logik nicht, weil die Äquivalenz hier durch den Rekurs auf Wahrheitswerte definiert wird. Im Zuge der unkritischen Übernahme des Äquivalenzbegriffs in die Übersetzungstheorie wurde „die Vergleichsentität ausgetauscht und somit nicht nur die Definition, sondern auch die gesamte Problemlage geändert" (Siever 2010: 83). Diese Änderung macht es notwendig, das Verhältnis von Sprache und Welt vom erkenntnistheoretischen Standpunkt zu reflektieren, weil, laut Siever, dessen „spezifische Konzeption“ für das Verhältnis von Text und Bedeutung entscheidend ist. In der traditionellen Konzeption des Übersetzens wird die Bedeutung als ein den Texten bzw. Wörtern inhärentes Merkmal aufgefasst. Aufgrund dieses Merkmals kann dem Ausgangs- und dem Zieltext die gleiche Bedeutung zugeschrieben werden. Siever weist darauf hin, dass zum Beispiel für Diller und Kornelius Äquivalenz „ein handliches Synonym für Bedeutungsgleichheit ist“ (2010:84). Somit genießt die Bedeutung in der traditionellen Auffassung einen objektiven Status, d. h. dass sie interpretationsunabhängig konzipiert wird. Im Rahmen des linguistischen Paradigmas wird deshalb „von der nützlichen Annahme ausgegangen, dass jeder Text einen objektiv gegebenen, $d$. h. unabhängig 
von allen konkreten Rezeptionsakten bestehenden Sinn aufweist" (Albrecht 1990: 71). Objektivität wird im Sinne von „Ausschaltung der Subjektivität“ verstanden (Hartfiel and Hillmann 1982: 646), d. h. „unabhängig vom Subjekt, seiner Wahrnehmung, Meinung und Wertung" (Hoffmeister 1955: 439). Dies steht jedoch im krassen Widerspruch zu den Grundannahmen der Linguistik von Saussure, wonach „die Einlösung des Sinns eines Zeichens interpretationsabhängig sei und mithin nur hypothetisch gelingen könne" (Frank 1991: 61). In der Tat stellt Saussure fest, dass „eine grammatische Tatsache (...) das Produkt einer Auslegung ist“ ${ }^{“ 6}$ (1957:100). Das Konzept einer „objektiven“, d. h. von jeder Interpretation unabhängigen Bedeutung wird demzufolge auch von Saussure verworfen. Wie dies von Siever hervorgehoben wird, Bedeutung wird vielmehr als ,ein Resultat komplexer (...) kognitiver Prozesse gedeutet“" (2010:84). Somit ist Sinn ,immer ein individuell konstruierter Sinn, der durch den Akt der Zeichensynthesis entsteht" (Siever 2010: 85). Dies deckt sich mit den erkenntnistheoretischen Feststellungen von Alfred Schütz, der darauf hingewiesen hat, dass der Sinn als Ergebnis des Interpretationsprozesses zunächst als subjektiver Sinn konstituiert, bevor er in der Interaktion von den Kommunikationspartnern intersubjektiv ausgehandelt wird (2004a).

In der weiteren Entwicklung der Übersetzungswissenschaft wurde der Begriff der Äquivalenz immer weiter ausdifferenziert und zur Bezeichnung verschiedener Phänomene verwendet. Darbelnet und Vinay in ihrer „Stylistique comparée du français et de l'anglais" (1958) bezeichnen damit eines der vier nicht wörtlichen Übersetzungsverfahren. Die Äquivalenz hat hier eine ganz andere Bedeutung als in den linguistischen Übersetzungstheorien. Auch die Verwendung des Äquivalenzbegriffs ist von Sprache zu Sprache unterschiedlich. So ist sein Gebrauch im Französischen, Deutschen und Englischen nicht gleichwertig.

Das Fehlen einer allgemeingültigen Definition der Äquivalenz in der Übersetzungstheorie und die erhebliche Ausdifferenzierung des Begriffs haben letztendlich zu einer ausgeprägten Unschärfe geführt (Schlicht von Rabenau 2016: 350). Der Grund dafür liegt im Exzess an Analyse. Günter Abel verwendet für dieses Phänomen den prägnanten Ausdruck „TausendfüßlerSyndrom“. Damit ist gemeint, dass der Erklärungswert eines Begriffs durch ein Übermaß an Analyse gemindert wird, was zu einer Art Paralyse führt (2016:168). Der Substanzverlust und die Unschärfe des Äquivalenzbegriffs

\footnotetext{
${ }^{6}$ Unsere Übersetzung der Originalpassage: „un fait grammatical (...) est pur produit d'une interprétation“.
} 
haben zur harschen Kritik in der Literatur geführt. Zahlreiche Autoren vertreten die Auffassung, dass dieser Begriff ungeeignet ist, um den Übersetzungsprozess im Sinne einer Entscheidung zwischen verschiedenen zur Wahl stehenden Alternativen angemessen zu beschreiben und bezeichnen ihn als eine Illusion (Snell-Hornby 1988: 13, Stolze 1982: 384), verwirrungsstiftend (Kussmaul 1986: 225) und sogar als „dead duck“ (Newmark 1981: X).

Außerdem wurde mit Blick auf den Äquivalenzbegriff der Vorwurf erhoben, den Eindruck einer linearen Übertragung von Sprache zu Sprache, d. h. der Isomorphie semantischer Systeme zu erwecken und die Dynamik des Übersetzungsprozesses zu verkennen.

Im Fazit kann festgehalten werden, dass das Äquivalenzparadigma im Sinne einer „linearen Übertragung der Bedeutung zwischen den Sprachen und Kulturen“ gescheitert ist (Renn 2006: 157).

\subsection{Gründe für das Scheitern des Äquivalenzbegriffs in der Übersetzungstheorie}

Zur Erklärung für das Scheitern des Äquivalenzbegriffs in der Übersetzungstheorie können mehrere Gründe angeführt werden. Einer von ihnen liegt darin, dass Äquivalenz ein inadäquater Begriff ist. Die Inadäquanz resultiert daraus, dass dieser Begriff das von Schütz mit Blick auf die Methodologie der Geisteswissenschaften aufgestellte Postulat der Adäquanz nicht erfüllt. Nach Schütz setzt Adäquanz voraus, einen Begriff in der Weise zu konstruieren, dass er vernünftig und verstehbar ist und zwar nicht nur für denjenigen, der ihn konstruiert, sondern auch für alle anderen:

Jeder Ausdruck, das in einem wissenschaftlichen System benutzt wird, muss so konstruiert werden, dass eine Handlung innerhalb der Lebenswelt [...] sowohl für den Handelnden als auch für seine Mitmenschen vernünftig und verstehbar ist (Schütz 2010: 226).

Allerdings ist dies in der Übersetzungstheorie nicht der Fall, weil Äquivalenz ein verzerrter Begriff ist. Die Verzerrung ergibt sich daraus, dass die Äquivokationen des logischen Begriffs der Äquivalenz in der Übersetzungstheorie bestehen bleiben, sodass die bilaterale Implikation weiter seinen Bedeutungsgehalt mitbestimmt. Bei der Übernahme des Begriffs der Äquivalenz von der Logik in die Übersetzungswissenschaft sind die dadurch eingetretenen Sinnmodifikationen nicht berücksichtigt worden. Eine Kontrol- 
le derartiger Sinnveränderungen bzw. Sinnverschiebungen ist jedoch nach Schütz unabdingbar, wenn vermieden werden soll „(...) naiv Begriffe und Sätze (...) zu übertragen, deren Gültigkeit wesentlich auf eine bestimmte Ebene beschränkt ist, d. h. auf deren implizite Voraussetzungen“" (2010:207). Ein weiterer Grund, weshalb die Äquivalenz ein ungeeigneter Begriff für die Übersetzungstheorie ist, liegt in der fehlenden Berücksichtigung der Dichotomie zwischen objektiver Bedeutung und subjektiver bzw. okkasioneller Bedeutung sprachlicher Ausdrücke (Schütz 2004a: 256). Schütz nimmt diese Unterscheidung im Anschluss an Husserl ${ }^{7}$ vor. Die objektive Bedeutung bzw. der objektive Sinn verweist auf das mit dem Ausdruck Bezeichnete und zwar unabhängig von dem Zusammenhang, in dem der Ausdruck gebraucht wird und von der Person, die ihn gebraucht (2004a: 256-257). Ausdrücke im Wörterbuch sind ein Beispiel für die objektive Bedeutung. Für Schütz haben aber alle Ausdrücke neben ihrer objektiven Bedeutung regelmäßig einen subjektiven und okkasionellen Sinn, den er als „Nebensinn“ bezeichnet. Dieser Nebensinn „umkleidet den objektiven Sinn des Zeichens, der den identischen Kern bildet“. Der Nebensinn besteht aus einer subjektiven Komponente. Dies ist der subjektive Sinn, d. h. die besondere Verwendungsweise, in der ein Ausdruck von einem Individuum oder von einer Gruppe verwendet wird. Ferner besteht der Nebensinn aus einer okkasionellen Bedeutung des Ausdrucks, die aus dem Zusammenhang, in dem er gebraucht wird, abgeleitet wird (2004a: 256). Diese Dichotomie ist der Grund für das grundsätzliche Fehlen von Äquivalenten bzw. dafür, dass lediglich Teiläquivalente von Ausdrücken existieren und somit auch ein Grund für die Polysemie. Diese Dichotomie bewirkt auch die Polarität zwischen objektivem und subjektivem Sinn (Schütz 2004a: 272), die ein heuristisches Prinzip der Sinndeutung und somit auch ein wesentlicher Grundsatz der Wissenschaften der Interpretation, insbesondere der Hermeneutik, der Philologie und der Rechtswissenschaft ist. Die Nichtberücksichtigung der Sinnpolarität hat die Unmöglichkeit zur Folge, bei der Interpretation eines Ausdrucks oder eines Textes die einschlägigen Auslegungsmuster beim Leser zu aktivieren. Wie wir im Weiteren sehen werden, hat die Relevanz sowohl mit der objektiven Bedeutung als auch mit der subjektiven, d. h. mit der individuell bedingten bzw. gruppenspezifischen und mit der okkasionellen, d.h. gebrauchsbedingten Bedeutung zu tun. Dies erscheint konsistent mit der sog. „Gebrauchstheorie“ der Bedeutung nach Wittgenstein (Loppe 2010) und zwar sowohl mit Blick auf die Bedeutung von

7 Zum Begriff der okkasionellen Zeichen: Edmund Husserl, Logische Untersuchungen I, [w:] Husserliana Bd. XIX / 1, Hg. v. Ursula Panzer, Den Haag: Nijhoff 1984, § 26, S. 85-92. 
Ausdrücken als auch auf die Bedeutung von Sätzen. Wittgenstein hat immer wieder auf diesen Aspekt der Bedeutung hingewiesen. So zum Beispiel in seinen Philosophischen Untersuchungen (PU $₫ 43$ ), wenn er feststellt, dass „die Bedeutung eines Wortes [...] sein Gebrauch in der Sprache [ist]“ (2015a:40) oder in seinem posthum erschienen Werk Über Gewissheit, wenn er beispielweise in der Bemerkung Nr. 61 feststellt: „(...) Eine Bedeutung eines Wortes ist eine Art seiner Verwendung“ (2015b:24) oder in Nr. 10 „(..) Nur im Gebrauch hat der Satz Sinn“ (2015b:11). Nach Schlicht von Rabenau wurde die Rolle des Gebrauchs hinsichtlich der Bedeutung bereits von Leibniz erkannt, der die Auffassung verfocht, „die Bedeutung sprachlicher Ausdrücke liege nicht in „Ideen“, die von den Ausdrücken repräsentiert würden, sondern im Gebrauch der von ihnen gemacht werde" (2014:11).

In dem vorliegenden Aufsatz wird die Auffassung vertreten, dass der Begriff der Relevanz es ermöglicht, zu bestimmen, inwiefern Übersetzungsentsprechungen geeignet sind, den Sinn des Ausgangstextes im Wege der Auslegung im Zieltext herzustellen, d. h. beim Leser die einschlägigen Deutungsmuster zu aktivieren, um ihn in die Lage zu versetzen, die korrekten Assoziationen zu machen, und damit die Orientierung in einer fremden Rechtsordnung zu gewährleisten. Soweit sich der Begriff der Äquivalenz letztendlich als ungeeignet erwiesen hat, um als Paradigma in der Übersetzung zu dienen, stellt sich die Frage nach einem neuen Paradigma und danach, welcher Begriff in Frage käme. Unseres Erachtens könnte der Begriff der Relevanz für die Übersetzung fruchtbar gemacht werden.

\section{Der Begriff der Relevanz}

\subsection{Vorbemerkungen}

Etymologisch wird der Ausdruck „Relevanz“ aus dem Latein von zwei verschiedenen Verben abgeleitet. Im Französischen („pertinence“) leitet er sich von dem Zeitwort „pertenere“, insbesondere von dessen unpersönlicher Form „pertinet“, die auf das, was wichtig bzw. von Belang ist, verweist. Im Deutschen und im Englischen hingegen leitet sich „Relevanz" von dem Zeitwort „relevare“ ab, das ebenfalls die Verweisung auf das, was wichtig ist, enthält. So ist es bereits die Etymologie, die auf die Verzahnung der Relevanz mit der Wichtigkeit verweist und die Aussage von Sandra Laugier: „ohne Belang, keine Relevanz" (2011:239) ${ }^{8}$ bekräftigt. Von Belang in einer Situation ist nach

\footnotetext{
${ }^{8}$ Unsere Übersetzung der Originalpassage: « Pas de pertinence sans importance ».
} 
Stegmaier „das Bedeutsame, das die Aufmerksamkeit fokussiert und zwingt, sich damit zu befassen“, wobei Situation mit der "Sachlage" identifiziert wird und verweist auf das, „womit man zurechtkommen muss“ (2008:153).

An dieser Stelle erscheint es nützlich, kurz auf den Begriff der Situation einzugehen, da er für das Phänomen der Relevanz nicht unbedeutend ist. Das Wort "Situation“ ist ebenfalls eine Entlehnung aus dem Lateinischen, nämlich von „situs" und bedeutet „Lage“. Die Erweiterung seines Gebrauchs hat dazu geführt, dass damit schließlich „Sachlage“, „Zustand“ oder „Lage der Dinge“ bezeichnet wird (Stegmaier 2008: 151).

Für Alfred Schütz, dessen Begriff der Relevanz Gegenstand der nachfolgenden Ausführungen sein wird, ist der Begriff der Situation ebenfalls sehr wichtig. Der Grund dafür liegt darin, dass zwischen Situation und Sprache ein Reziprozitätsverhältnis besteht, insofern als „die Situation die Sprache bestimmt und die Sprache die Situation bestimmt" (Schütz 2003b: 246). Das bedeutet, dass die Sprache gebraucht wird, um die Situation zu definieren und ihrerseits wiederum ein Element der Situation ist. Nach Schütz wird die Sprache durch folgende Elemente der Situation bestimmt: gemeinsame Umgebung, Typisierung und Relevanz (2003b:248). Außerdem sind nach Schütz sowohl die thematische als auch die Interpretationsrelevanz „situational bedingt" (2004b:105). Dies ist der Grund, weshalb es keine thematische und keine Auslegungsrelevanz per se gibt und dass es unabdingbar ist, die Modifikationen zu beachten, die sich aus den Umständen der jeweiligen Situation ergeben (2004b:105). Daraus resultiert unseres Erachtens die grundlegende Bedeutung der Situation für die Übersetzung. Denn die Verwobenheit zwischen Sprache, Denken und Handeln wird eben in der Situation deutlich (Humboldt 1903-1936, Bd. 7, T. 1; S. 53). Die Situation bildet den pragmatischen Kontext im weitesten Sinne, in den eine Übersetzung eingebettet ist, wobei der Begriff der Situation aufgrund der Spezifik des Übersetzungsprozesses nicht nur die Produktionssituation, sondern auch bzw. vor allem die Verwendungssituation einer Übersetzung erfasst.

\subsection{Der Begriff der Relevanz nach Alfred Schütz}

Alfred Schütz war ein österreichischer Jurist, Philosoph und Soziologe (18991959). Er hat das Phänomen der Relevanz systematisch in seinem Hauptwerk aus dem Jahre 1947 „Das Problem der Relevanz" erforscht. Dieses Werk ist posthum erstmals 1970 in englischer Sprache unter dem Titel „Reflections on the Problem of Relevance" erschienen. Für unsere Untersuchungen wird die neue Auflage in deutscher Sprache mit dem Titel „Relevanz und Han- 
deln"verwendet (Schütz 2004b). Nach Saracevic stellt die Theorie von Schütz die umfassendste Theorie der Relevanz dar, die bisher vorgelegt worden ist (1996).

Für Schütz ist Relevanz „das (...) wichtigste und zugleich schwierigste Problem“ (2003a:253). Indem sie den Verlauf der Auslegung steuert, spielt die Relevanz eine wesentliche Rolle bei der Konstitution von Sinn, wobei Sinn als das Ergebnis der Auslegung definiert wird (2003a:184). Der Begriff des Sinns wurde ursprünglich von Max Weber geprägt (1976). Das Vorhaben von Schütz bestand darin, diesen Begriff epistemologisch zu fundieren (2004b:14). Schütz hat von der Relevanz eine Reihe von Definitionen gegeben, die deren dynamischen Charakter zum Ausdruck bringen. Für die vorliegende Analyse wird lediglich auf diejenigen Definitionen zurückgegriffen, die uns am prägnantesten erscheinen. Das Problem der Relevanz wird von Schütz beschrieben als:

- Ein Grundphänomen: das Haben von Sinn (2004b:51).

- Die „Auswahl, die es ermöglicht, aus der Totalität der Welt' bzw. aus einem verfügbaren Sinnuniversum bestimmte Sinngehalte herauszugreifen.", d. h. die Selektion bedeutsamer Inhalte (2004b:51).

- Die Relation im Prozess des Verstehens: „Verstehen heißt Relevanzrelation feststellen" (2004b:47).

- Die Relevanz : „Zentralbegriff der Geisteswissenschaften“ (2004b:51).

- Im Recht: „Tatbestände, an die sich keine Norm knüpft, sind irrelevant" (2004b:46); konsequenterweise folgt daraus a contrario, dass relevant bzw. rechtserheblich allein Tatbestände sind, die an eine Norm geknüpft werden.

- Schließlich ist Relevanz für Schütz ein Korrelat des Sinns insofern als „nur zwischen Relevantem Sinnzusammenhänge bestehen und nur das im Sinnzusammenhang Stehende ist relevant" (2004b:49).

Die zitierten Definitionen zeigen, dass der Begriff der Relevanz bei Schütz stets an den Begriff des Sinns gekoppelt ist und als der Grundmechanismus der Selektivität beschrieben wird, der den Prozess der Sinnkonstitution leitet (2011:11). Die von Relevanz gesteuerte Auswahl von mit Sinn behafteten Elementen ist, wie die Semiose, ein unendlicher Prozess. Sie ist „immer am Werk (...) und eben deshalb ist Relevanz ein zentraler Begriff der (...) Geisteswissenschaften“ (2004b:15). Auch die Übersetzungstheorie kann mithin nicht davon absehen, den Begriff der Relevanz zu untersuchen und zu versuchen, ihn für ihren Gegenstand, d. h. für die Anfertigung von Übersetzungen, fruchtbar zu machen. 


\subsubsection{Typologie der Relevanz}

In der von ihm vorgeschlagenen Typologie unterscheidet Schütz drei Arten von Relevanz, nämlich: thematische Relevanz, Interpretationsrelevanz und Motivationsrelevanz. Alle drei Relevanztypen bestehen nicht unabhängig voneinander, sondern bilden ein durch Interdependenz geprägtes System, in dem sie sich gegenseitig beeinflussen und aufeinander beziehen, denn: „Es gibt keine isolierte Relevanz. Relevanzen, welcher Kategorie auch immer, sind stets in Systemen gruppiert und untereinander verbunden (...) "Schütz 2004b: 104). Das Relevanzsystem bildet den Bezugsrahmen für die Auslegung (Schütz 2003b: 191).

1) Thematische Relevanz

Von allen Relevanzarten ist die thematische Relevanz die wichtigste, weil sie ubiquitär und dem Thema inhärent ist. Sie verweist auf den objektiven Aspekt der Relevanz. Unter dem Stichwort „topicality“ wurde die thematische Relevanz in den Information Sciences als die Grundvoraussetzung der Relevanz beschrieben (Greisdorf 2003). Dies ist auch das einzige Kriterium, über das ein Konsens in der Literatur auf diesem Wissensgebiet besteht, während alle anderen Kriterien kontrovers diskutiert werden (Xu and Chen, 2006). Der Begriff „Thema" wird hier in der allgemeinen Bedeutung verstanden und mit dem Textinhalt identifiziert (Hoffmann, 2000). Bei Schütz verweist er auf ein zu lösendes Problem (2004b:189). Thematisch ist somit das, was problematisch ist und die Aufmerksamkeit fokussiert. Mit Blick auf die Übersetzung würde dies heißen, dass das Thema auf Folgendes verweist:

a) Auf die anzufertigende Übersetzung als solche, d. h. als ein zu lösendes Problem,

b) Auf den Textinhalt,

c) Auf das, was von dem jeweiligen Wissensgebiet (Recht, Medizin, etc.) vorgegeben wird,

d) Auf das, was in dem jeweils zu übersetzenden Text mit der Auswahl der geeigneten Termini zu tun hat (Bsp. „Herausgabe $\rightarrow$ restitution bzw. revendication“ oder „Verbindung $\rightarrow$ jonction vs. accession mobilière“).

2) Interpretationsrelevanz Jedes Thema bedarf einer Interpretation. Das bedeutet, dass es in die vorhandenen Deutungsschemata eingeordnet werden muss. Erst die Interpretation verleiht den Sinn. Für Schütz „Sinn ist (...) keine Eigenschaft (...), sondern das Ergebnis der Interpretation einer vergangenen Erfahrung" (2003: 184). 
Die Interpretation, d. h. die Subsumption der neuen thematischen Elemente unter die vorhandenen Deutungsschemata wird durch die Interpretationsrelevanz ermöglicht. Allerdings werden bei der Subsumption nicht alle im Wissensvorrat vorhandenen Deutungsschemata verwendet, sondern nur diejenigen, mit denen das auszulegende Thema verglichen werden kann (Schütz 2004b: 98). Das Deutungsschema wird als Sedimentierung aller früheren Interpretationen definiert. Es entsteht im Wege der "Sedimentierung von Sinn" (Schütz 2003a: 357) bzw. der "Sedimentierung von Bedeutungen“ (Schütz 2003a: 255). Die Deutungsschemata sind zum einen durch die jeweilige Kultur und Sprache bedingt und als solche dem Individuum ontologisch vorgegeben. Jede Kultur und jede Sprache bildet somit ein System von Interpretationsrelevanzen, die den Bezugsrahmen für individuelle Auslegung darstellen (Schütz 2004a: 119) Zum anderen sind die kultur-, sprach- und gruppenspezifischen Deutungsschemata erlernbar. Das bedeutet, dass die für die Auslegung relevanten Elemente gelernt werden müssen (Schütz 2004b: 105).

3) Motivationsrelevanz

Die dritte Art der Relevanz ist die Motivationsrelevanz. Sie verweist auf die Wichtigkeit einer Auslegungsentscheidung. Für Schütz besteht „die Wichtigkeit, richtig (...) auszulegen, in der Tatsache, dass nicht nur die zu wählenden Mittel, sondern auch die zu erreichenden Ziele" von dieser Entscheidung beeinflusst werden (2004b:107). Die Motivationsrelevanz hat demzufolge damit $\mathrm{zu}$ tun, was von Belang ist. Jede Interpretation wird durch ein Interesse in Gang gesetzt. Der Begriff „Interesse“ bezeichnet „ein System der Motivationsrelevanzen, die die Auswahltätigkeit meines Bewusstseins leiten" (2004b:122). Die Auswahl bzw. die Selektion ist der Grundmechanismus im heuristischen Prozess, wie dies sowohl durch die Recherchen in den Information Sciences (Saracevic, 1996) als auch durch die Neuroanatomie der Sprache bestätigt wird: „Jedem Diskurs muss eine selektive Auswahl der mitzuteilenden Information vorausgehen "99 (Petrides 2014: 174). Die Relevanzen spielen dabei eine wesentliche Rolle, weil das komplexe System, das sie bilden, die Selektion der zur Auswahl stehenden Elemente oder der konkurrierenden Interpretationen leitet.

${ }^{9}$ Unsere Übersetzung der Originalpassage: "All discourse must be preceded by selective retrieval of the information to be articulated". 


\subsubsection{Wissensvorrat und Relevanz}

Der Wissensvorrat wird als Vorrat an Auslegungsschemata bzw. als ein individueller Wissens- und Erfahrungsbestand definiert (Schütz 2003b: 356). Der Wissensvorrat ist für die Konstitution von Sinn insofern wesentlich, als der Sinn interaktiv unter Bezug auf die Ressource „Wissen“ konstituiert wird (Endress 2006: 50). Der Wissensvorrat stellt den Bezugsrahmen dar, in dem jegliche Interpretation stattfindet. Er ist somit auch für die Relevanz wesentlich, weil die Relevanz innerhalb dieses Bezugsrahmens operiert. Der Wissensvorrat besitzt eine Reihe von Merkmalen, von denen hier nur die wichtigsten erwähnt werden. Der Wissensvorrat ist durch eine besondere nach Bekanntheitsgraden strukturierte Organisationsform charakterisiert (Schütz 2003a: 357). Ferner ist der Wissensvorrat historisch, weil jeder seiner Elemente auf frühere Erfahrungen bezogen ist (Schütz 2003b: 255). Die weiteren Merkmale des Wissensvorrats sind: Kultur-, Sprach- und Gruppenspezifik, Verfügbarkeit, Erwerb durch Lernen, Inhomogenität, stetige Veränderbarkeit sowohl hinsichtlich des Umfangs als auch der Struktur, pragmatische Bestimmtheit. Aus Platzgründen wird von der Erläuterung dieser Merkmale in dem vorliegenden Aufsatz abgesehen. Der Wissensvorrat hat eine doppelte Funktion: zum einen bildet er ein gemeinsames Interpretationsschema und dient der gegenseitiger Verständigung (Schütz 2003a: 330) und zum anderen fungiert er als das Bindeglied zwischen Handeln und Wissen (Schütz 2011: 17). Auch eine Wissenschaft kann als „ein Analogon unseres verfügbaren Wissensvorrats interpretiert werden" (Schütz 2003a: 273). In diesem Sinne stellt sie „ein Korpus von untereinander verbundenen, verifizierbaren und geprüften Aussagen dar, die ein Optimum an Klarheit, Deutlichkeit und Konsistenz aufweisen (...)" (2003a: 273). Der wissenschaftliche Wissenskorpus ist außerdem nach den für die jeweilige Wissenschaft geltenden Kriterien und Methoden konstituiert. Er ist homogen, anonym und von den individuellen Relevanzsystemen unabhängig, was seine Homogenität gewährleistet (2003a: 273).

\subsubsection{Voraussetzungen der Kommunikation}

Nach Schütz muss jede Kommunikation zwei wesentliche Voraussetzungen erfüllen, damit sie erfolgreich wird und die Verständigung gewährleistet. Die erste Voraussetzung besteht in der Vertauschbarkeit der Perspektiven der Kommunikationspartner. Das bedeutet, dass jeder von ihnen, in der Lage 
sein soll, ein Problem von der Perspektive des anderen zu betrachten. Die zweite Voraussetzung besteht in der Kongruenz der Relevanzsysteme. Das bedeutet, dass zumindest die thematische und die Interpretationsrelevanz eine ähnliche Struktur haben muss, damit die Verständigung gelingt (2003a:342). Dies setzt voraus, dass im Wissensvorrat der Kommunikationspartner mindestens ähnliche wenn nicht identische Auslegungsschemata vorhanden sein müssen.

Betrachtet man nun die Praxis der Rechtsübersetzung aus diesem Blickwinkel, wird der Grund für den Misserfolg der Kommunikation erkennbar: es ist schwierig für den Übersetzer, die von Schütz definierten Voraussetzungen einer erfolgreichen Kommunikation zu erfüllen. Denn der Übersetzer und der Jurist können ihre Perspektiven nicht vertauschen: was für den Übersetzer wichtig oder problematisch und damit relevant ist, ist nicht unbedingt relevant für den Juristen und umgekehrt. Ferner besteht keine Übereinstimmung oder Ähnlichkeit des Wissensvorrats und der Auslegungsschemata des Übersetzers und des Juristen weder in der Ausgangs- noch in der Zielsprache. In der Tat werden beide von unterschiedlichen Relevanzen geleitet sowohl im Hinblick auf den individuellen als auch disziplinspezifischen Wissensvorrat. Schwierig heißt aber nicht unmöglich. Die Möglichkeit der Verständigung in der Rechtsübersetzung wird dadurch gewährleistet, dass sich der Übersetzer die für den Juristen in der Ausgangs- und der Zielsprache jeweils gültigen Relevanzsysteme so weit wie möglich durch Lernen aneignet. In der Rechtsübersetzung besteht die Aufgabe des Übersetzers unseres Erachtens darin, die Relevanzstrukturen so herzustellen, dass die unvertrauten Elemente des fremden Rechts bis zu einem gewissen Grad an die vertrauten Elemente des für den Juristen heimischen Rechts herangeführt werden, ohne den Eindruck der Isomorphie zwischen Rechtsordnungen zu erwecken, gleichzeitig aber die Orientierung im fremden Rechtssystem zu ermöglichen. Was Orientierung bedeutet und wie sie praktisch durch relevante Übersetzung von Rechtsinstituten zu erreichen ist, wird im Weiteren erläutert und anhand einiger Beispiele aus der Übersetzungspraxis veranschaulicht. Grundvoraussetzung hierfür ist aber, dass man in der Rechtsübersetzung von der Treue zum Original abrückt und dem Übersetzer eine echte Freiheit in der kreativen Lösung der sich stellenden Übersetzungsprobleme zuerkennt. Wie Wolff feststellt, ist dies gegenwärtig bei Weitem nicht der Fall, weil die Bedeutung als „in Stein gemeißelt“ betrachtet wird, sodass die Forderung nach Kreativität in der Rechtsübersetzung auf erhebliche Zurückhaltung, ja gar auf Ablehnung stößt. Wolff weist darauf hin, dass viele Autoren, darunter insbesondere Šarcevic, fordern, Kreativität in der Rechtsübersetzung „müsse 
auf ein „zulässiges Mindestmaß“ reduziert werden (2011:229). Diese Forderung hat ihren Ursprung in der Vorstellung, dass Kreativität eine Gefahr für die einheitliche Auslegung und Anwendung des fraglichen Ausgangs- und Zieltextes" ${ }^{\text {"10 }}$ sei (Sarcevic 1998: 289). Wolff verficht jedoch die Auffassung, dass es in der Rechtsübersetzung vielmehr darauf ankomme: „(...) juristische Übersetzungen mit natürlicher Ausdrucksweise [zu produzieren], die es ermöglichen, die sozialen und rechtlichen Belange besser zu beachten, als sich lediglich auf den toten Buchstaben des Gesetzes zu konzentrieren "11 (2011:230). Dieser Auffassung schließen wir uns insofern an, als diese Art von Rechtsübersetzung, die den Leser im Blick behält, geeignet ist, ihm die Orientierung in einer fremden Rechtsordnung zu ermöglichen.

\section{Relevanz und Orientierung}

Der Terminus „Orientierung “ besitzt eine allgemeine und eine philosophische Bedeutung. Er entstammt der Geographie und wird von dem lateinischen Partizip Präsens ,oriens', d. h. ,sich erhebend' abgeleitet. Seine ursprüngliche Bedeutung ist „sich dem Osten zuwenden“. Der Orient ist [...] das Land in Richtung der aufgehenden Sonne (,sol oriens'). Nach Kant bedeutet „sich orientieren“ in der eigentlichen Bedeutung des Wortes: „aus einer gegebenen Weltgegend [...] die übrigen, namentlich den Aufgang zu finden" (Stegmaier 2008: 55). Auf das Gebiet der Erkenntnis übertragen bedeutet der Terminus nach Wilhelm Traugott Krug „sich auf jenem Gebiet zurechtfinden“ (Stegmaier 2008: 59).

In seiner philosophischen Bedeutung wird „Orientierung " folgendermaßen definiert: „Orientierung ist eine Leistung, sich in einer Situation zurechtzufinden“ (Stegmaier 2008: 2). Nach Stegmaier weist die Orientierung im philosophischen Sinne die Merkmale der Zeitlichkeit und der Anschlussfähigkeit auf. Zeitlichkeit bedeutet, dass die Orientierung mit jeder neuen Situation wechselt. Deshalb könne sie nicht abschließend, sondern nur "par provision" definiert werden (Stegmaier 2005: 17). Die Abhängigkeit der Orientierung von der Zeit kommt am deutlichsten in der Rede vom ,Halt ' der Orientierung zum Ausdruck (Stegmaier 2016: 49). Die Anschlussfähigkeit

${ }^{10}$ Die Originalpassage lautet: „(...) poses a threat to the uniform interpretation and application of the source and target text in question”.

${ }_{11}$ Die Originalpassage lautet: „natural legal translations that better respect the sociolegal significance of the text rather than the 'dead letter of the law' (...)". 
der Orientierung verweist darauf, „was jemand mit einer Situation anfangen kann und was er aus ihr lernen kann" (Stegmaier 2008: 22).

Übertragen auf die Übersetzungswissenschaft wird man sagen können, dass wenn die Aufgabe der Rechtsübersetzung darin besteht, dem Rechtsexperten die Orientierung in einer fremden Rechtsordnung zu ermöglichen, so kann diese Orientierung nur vorläufig sein, weil sie von der jeweiligen und immer neuen Übersetzungssituation abhängt. Die Übersetzung als Text könnte in einer fremden Rechtsordnung dann als anschlussfähig bezeichnet werden, wenn der Leser daraus etwas lernen kann oder, wenn er sie in einer bestimmten Situation verwenden kann. Die Relevanz und damit auch die Anschlussfähigkeit der Übersetzung als Text hängen unseres Erachtens nicht damit zusammen, ob der Übersetzer in der Lage ist, in seinem Zieltext das von Šarcevic verfochtene Postulat der „Äquivalenz der Rechtswirkungen“ zu erfüllen. Dieses Postulat ist ohnehin eine „praktische Unmöglichkeit“" (Wolff 2011: 238). Darauf kommt es auch nicht an, weil der Übersetzer keine rechtlich erhebliche Qualifikation vornimmt. Worauf es hingegen in der Rechtsübersetzung ankommt, ist, vermittels semantischer Analyse die Orientierung zu ermöglichen und somit die Richtung aufzuzeigen, in der sich „der Sinn des Textes erschließt“ (Ricoeur 1986: 156). Damit werden die einschlägigen Auslegungsschemata beim Leser aktiviert. Orientierung und Relevanz sind deshalb grundlegende Begriffe für die Rechtsübersetzung, weil sie der in der Vielfalt des Sprachgebrauchs wurzelnden Relativität der Rechtsbegriffe (Felix 1998: 211) entgegenwirken.

\subsection{Relevanz und Orientierung: Korrelativa}

Relevanz und Orientierung korrelieren miteinander insoweit als ihr Grundmechanismus in der Selektion besteht. Beide Begriffe haben mit der Ausrichtung der selektiven Aufmerksamkeit zu tun und sind in die Entscheidungen bei konkurrierenden Interpretationen involviert.

Relevanz und Orientierung korrelieren aber auch insoweit miteinander, als sie beide Korrelate der Situation sind. Die Relevanz bedingt die Auswahl der Elemente, die in einer Situation von Belang sind. Der Sinn wird durch die Einordnung dieser Elemente in einen durch die Situation bestimmten $\mathrm{Zu}$ sammenhang konstituiert, Sodass ein Sinnzusammenhang entsteht. Die Entstehung eines Sinnzusammenhangs ermöglicht dann die Orientierung in der jeweiligen Situation. Relevant für eine Situation ist das, „womit man sich je nach der jeweiligen Interessenlage auseinandersetzen muss" (Schütz, 2003b). 
Schließlich ist Relevanz insofern mit der Orientierung verzahnt und für sie auch grundlegend, als sie „die Unterscheidung des Unterschieds, der einen Unterschied macht ${ }^{\text {“12 }}$, d. h. die Unterscheidung dessen, was von Belang ist, ermöglicht (Stegmaier 2008: 158).

\section{Die Bedeutung der Relevanz in der Rechtsübersetzung anhand von Beispielen aus der Praxis}

Was die Rede von der Relevanz und der Orientierung in der Praxis der Rechtsübersetzung bedeutet, soll hier anhand einiger weniger Beispiele aus der Übersetzungspraxis veranschaulicht werden. An erster Stelle möchten wir das Beispiel der Übersetzung des im deutschen Recht geläufigen Begriffs der Grundschuld ( $\$ 1191$ BGB) erörtern. Im Französischen wird dieser Begriff häufig mit „dette foncière“ übersetzt. Es handelt sich hier um eine wörtliche Übersetzung, die im französischen Recht keinen Bedeutungsgehalt aufweist. Für den Leser ist es schwierig, diesen Terminus in einen Sinnzusammenhang einzuordnen und damit etwas ,anzufangen“. Die Übersetzung ist somit weder anschlussfähig noch relevant. Es kommt aber auch vor, dass "Grundschuld“ auf Französisch mit „hypothèque“ [Hypothek] übersetzt wird. Auch dies ist irrelevant und nicht anschlussfähig. Denn ein Hauptmerkmal der Grundschuld ist ihr nicht akzessorischer Charakter. Das bedeutet, dass eine Grundschuld vom Bestand einer Forderung unabhängig ist. Dies ist auch der grundlegende Unterschied zu Hypothek. Grundschuld mit „Hypothek“ zu übersetzen, ist schlechthin irrelevant, weil diese Übersetzung von der Nichtakzessorietät als Grundmerkmal der Grundschuld völlig absieht, und diese sogar mit dem Rechtsinstitut der Hypothek verwechselt (die eben von dem Bestand einer Forderung abhängt). Unseres Erachtens würde es sich hier empfehlen, eine Übersetzung zu verwenden, die zwar allgemeiner ist, dafür aber den Kern der Sache trifft und somit auch den „Unterschied macht“. Eine solche Übersetzung könnte zum Beispiel lauten „sureté réelle immobilière sans caractère d'accessoriété“. Daraus folgt, dass eine Übersetzung dann relevant ist, wenn sie den Sinn des AT im ZT adäquat im Verhältnis zu einem wissenschaftlichen Korpus und zum Horizont des Rezipienten herstellt.

\footnotetext{
12 Der Begriff des „Unterschieds, der einen Unterschied macht“ stammt von Gregory Bateson: „A bit of information is definable as a difference which makes a difference" (Stegmaier 2008: 157, Fußnote 10).
} 
Als weiteres Beispiel kann die Übersetzung des Artikels 259 des schweizerischen Zivilgesetzbuchs angeführt werden. Diese Vorschrift mit dem Titel „Kauf bricht Miete“ („la vente rompt le bail“) regelt die Rechtsfolgen des Verkaufs eines vermieteten Grundstücks. Im deutschen Zivilrecht gibt es ebenfalls eine Vorschrift, die die Rechtsfolgen des Verkaufs eines vermieteten Grundstücks regelt ( $\$ 566 \mathrm{BGB})$, wobei der deutsche Gesetzgeber sich für die entgegengesetzte Lösung entschieden hat, nämlich „Kauf bricht nicht Miete“ („la vente ne rompt pas le bail"). Virgile Rossel, der Übersetzer des schweizerischen Zivilgesetzbuchs, hat die Vorschrift des Artikels 259 ZGB mit „Veräußerung des Mietgrundstücks“ („aliénation de l'immeuble loué“) übersetzt. Mit seiner Lösung abstrahiert Rossel vollkommen von der Anspielung auf das deutsche Recht, weil dieser Ausdruck für die französischsprachigen Juristen keinen Bedeutungsgehalt hat. Damit geht aber auch die Verweisung auf die Rechtsfolgen verloren, die in der Vorschrift enthalten ist. Weil sie zu vage ist, frustriert diese Übersetzungslösung die Erwartungen des Juristen und ist auch nicht geeignet, Orientierung zu ermöglichen. Mit Blick auf die vorstehenden Ausführungen erweist sich diese Übersetzungslösung als irrelevant.

Ein ähnliches Beispiel ist das Institut der Verbindung mit beweglichen Sachen im deutschen Recht $(\$ 947 \mathrm{BGB})^{13}$. Die französische Übersetzung hierfür lautet: ,jonction avec des choses mobilières“ (Lardeux et al., 2010:337). Abgesehen von der Tatsache, dass es sich auch hier um eine „formale Äquivalenz" handelt, lässt diese Lösung völlig außer Acht, dass das französische Recht das Institut der „accession mobilière“ ${ }^{14}$ kennt, das durchaus eine geeignete Entsprechung darstellen könnte.

\section{Fazit}

Der Begriff der Relevanz ermöglicht unseres Erachtens, die Natur der von Koller postulierten Relation zwischen Ausgangs- und Zieltext in der

${ }^{13}$ Diese Vorschrift lautet wie folgt: (1) Werden bewegliche Sachen miteinander dergestalt verbunden, dass sie wesentliche Bestandteile einer einheitlichen Sache werden, so werden die bisherigen Eigentümer Miteigentümer dieser Sache; die Anteile bestimmen sich nach dem Verhältnis des Wertes, den die Sachen zur Zeit der Verbindung haben. (2) Ist eine der Sachen als die Hauptsache anzusehen, so erwirbt ihr Eigentümer das Alleineigentum.

${ }^{14}$ Es handelt sich um Artikel 566 des französischen Zivilgesetzbuchs (Code civil), der folgenden Wortlaut hat: «Lorsque deux choses appartenant à différents maîtres qui ont été unies de manière à former un tout, sont néanmoins séparables, en sorte que l'une puisse subsister sans l'autre, le tout appartient au maitre de la chose qui forme la partie principale à la charge de payer à l’autre la valeur, estimée à la date du paiement, de la chose qui a été unie ». 
Übersetzung zu definieren. Allerdings erschöpft sich die Natur dieser Relation nicht in einer wie auch immer definierten Äquivalenz, sondern ist als ein dynamischer Prozess der Sinnkonstitution im Zieltext zu verstehen, wobei die Relevanz des konstruierten Sinns erst a posteriori beurteilt werden kann. Die Sinnkonstitution vollzieht sich in der Interaktion von folgenden Elementen: den von einem Wissensgebiet vorgegebenen thematischen und Interpretationsrelevanzen, dem subjektiven Relevanzsystem des Übersetzers und dem für den Juristen einschlägigen Relevanzsystem einer bestimmten Rechtsordnung. Ausschlaggebend für die Rechtsübersetzung ist demzufolge nicht nur die adäquate Wiedergabe der Rechtsterminologie, sondern vielmehr die Vermittlung des der jeweiligen Rechtsordnung zugrundeliegenden Relevanzsystems, das von dem zu übersetzenden Text getragen wird. Dies setzt voraus, dass mit Blick auf den Wissensvorrat des Lesers (Rechtsexperten) und auf das für ihn geltende Relevanzsystem übersetzt wird. Denn nur eine solche Übersetzung frustriert seine Erwartungen nicht. Praktisch heißt dies, dass "Verbindung“ eben nicht wortwörtlich mit „jonction“, sondern mit „accession mobilière“ und „Verarbeitung" nicht mit „transformation“, sondern mit „spécification“ zu übersetzen wären. Wir sehen allerdings, dass eine kreative Übersetzung, die auf den Sinn des juristischen Textes abstellt, die Beherrschung der thematischen und der Auslegungsrelevanzen sowie erhebliche Recherchen in der Fachliteratur voraussetzt. Ein solches Übersetzungsmodell ist dennoch keine Utopie, weil sowohl die thematischen als auch die Interpretationsrelevanzen durch Aneignung des juristischen Grundwissens und durch Erfahrung erlernt werden können. Der Vorteil, den dieses Modell bietet, besteht darin, dass es dem Leser die Orientierung in einem fremden Rechtssystem ermöglicht, ohne in die Falle einer exzessiven Nostrifizierung zu tappen. Im Fazit kann man feststellen, dass, wenn die Relevanz das Haben von Sinn ist, ist eine Übersetzung dann relevant, wenn sie in der gegebenen Situation für ihren Leser einen Sinn hat.

\section{Literatur}

Abel, G., 2016, Quellen der Orientierung. In: Bertino, A., Poljakova, E., Rupschus, A. \& Alberts, B. (eds.) Zur Philosophie der Orientierung. Berlin: de Gruyter.

Albrecht, J., 1990, Invarianz, Äquivalenz, Adäquatheit. In: Arntz Reiner \& Thome, G. (eds.) Übersetzungswissenschaft. Ergebnisse und Perspektiven. Tübingen. 
Catford, J., 1965, A linguistic theory of translation. An essay in applied linguistics., London, Oxford University Press.

Darbelnet, J. \& Vinay, J.-P., 1958, Stylistique comparée du français et de l'anglais, Paris, Didier.

Endress, M., 2006, Alfred Schütz, Konstanz, UVK Verlagsgesellschaft.

Felix, D., 1998, Einheit der Rechtsordnung. Zur verfassungsrechtlicher Relevanz einer juristischen Argumentationsfigur., Tübingen, Mohr Siebeck.

Frank, M., 1991, Selbstbewusstsein und Selbsterkenntnis, Stuttgart, Reclam.

Greisdorf, H., 2003, Relevance thresholds: a multi-stage predictive model of how users evaluate information. Information Processing \& Management, $39,403-423$.

Hartfiel, G. \& Hillmann, K.-H., 1982, Wörterbuch der Soziologie, Stuttgart, Kröner.

Hoffmann, L., 2000, Thema, Themenentfaltung, Makrostruktur. In: Brinker, K., Antos, G., Heinemann, W. \& Sager, S. F. (eds.) Text-und Gesprächslinguistik. Berlin. New York: Walter de Gruyter.

Hoffmeister, J., 1955, Wörterbuch der philosophischen Begriffe, Hamburg, Meiner.

Humboldt, W., Von, 1903-1936, Werke, Berlin, B. Behr.

Jakobson, R., 1959, On linguistics aspects of translation. In: Brower, R., A. (ed.) On translation. Cambridge, Massachussetts: Harvard University Press.

Kussmaul, P., 1986, Übersetzen als Entscheidungsprozess. Die Rolle der Fehleranalyse in der Übersetzungsdidaktik. In: Snell-Hornby, M. (ed.) Übersetzungswissenschaft. Eine Neuorientierung. Tübingen: Francke.

Lardeux, G., Legeais, R., Pédamon, M. \& Witz, C., 2010, Code civil allemand. Traduction commentée, Paris, Dalloz.

Laugier, S., Plaud, S., 2011, La Philosophie analytique, Paris, Ellipses Editions. Loppe, T., 2010, Bedeutungswissen und Wortgebrauch. Entwurf einer Semantik im Anschluss an Wittgenstein und Putnam, Tübingen, Narr Francke Attempto Verlag.

Newmark, P., 1981, Approaches to translation, Oxford, Pergamon Press.

Nida, E., A., 1964, Towards a science of translating, Leiden, E. J. Brill.

Petrides, M., 2014, Neuroanatomy of Language. Regions of the Human Brain, Elsevier.

Reiss, K., 1971, Möglichkeiten und Grenzen der Übersetzungskritik: Kategorien und Kriterien für eine sachgerechte Beurteilung von Übersetzungen, München, Hueber.

Renn, J., 2006, Übersetzungsverhältnisse. Perspektiven einer pragmatischen Gesellschaftstheorie, Weilerswist, Velbrück Wissenschaft. 
Ricoeur, P., 1986, De l'herméneutique des textes à celle de l'action. Du texte à l'action. Paris: Editions du Seuil.

Saracevic, T. Relevance reconsidered. Second Conference on Conceptions of Library and Information Science (CoLIS 2), 14-17 Oct.1996 1996 Copenhange. Information science, 201-218.

Sarcevic, S., 1998, Creativity in legal translation.

How much is too much? In: Chestermann, A., Salvador, N. G. S. \& Gambler, Y. (eds.) Translation in context. Selected Papers from the EST Congress Granada 1998. John Benjamins Publishing Company.

Saussure, F. D., 1957, Cours de linguistique générale (1908-1909): Introduction. Cahiers Ferdinand de Saussure, 15, 3-103.

Schlicht Von Rabenau, M., 2014, Der Philosophische Begriff des Gebrauchs. Platon, Kant, Wittgenstein, Münster, mentis Verlag.

Schlicht Von Rabenau, M., 2016, Kompetenz als Schlüsselbegriff der Bildungstheorie. Vorschlag einer orientierungsphilosophischen Fundierung. In: Bertino, A., Poljakova, E., Rupschus, A. \& Alberts, B. (eds.) Zur Philosophie der Orientierung. Berlin: De Gruyter.

Schütz, A., 2003a, Theorie der Lebenswelt 1. Zur pragmatischen Schichtung der Lebenswelt., Konstanz, UVK Verlagsgesellschaft.

Schütz, A., 2003b, Theorie der Lebenswelt 2. Zur kommunikativen Ordnung der Lebenswelt., Konstanz, UVK Verlagsgesellschaft mbH.

Schütz, A., 2004a, Der Sinnhafte Aufbau der sozialen Welt., Konstanz, UVK Verlagsgesellschaft.

Schütz, A., 2004b, Relevanz und Handeln 1. Zur Phänomenologie des Alltagswissens., Konstanz, UVK Verlagsgesellschaft mbH.

Schütz, A., 2010, Zur Methodologie der Sozialwissenschaften., UVK Verlagsgesellschaft $\mathrm{mbH}$.

Schütz, A., 2011, Relevanz und Handeln 2. Gesellschaftliches Wissen und politisches Handeln., Konstanz, UVK Verlagsgesellschaft.

Siever, H., 2010, Übersetzen und Interpretation. Die Herausbildung der Übersetzungswissenschaft als eigenständige wissenschaftliche Disziplin im deutschen Sprachraum von 1960 bis 2000, Frankfurt am Main, Peter Lang.

Snell-Hornby, M., 1988, Translation studies: An integrated approach, Amsterdam, John Benjamins Publishing Company.

Stegmaier, W., 2005, Orientierung. Philosophische Perspektiven, Frankfurt am Main, Suhrkamp Verlag.

Stegmaier, W., 2008, Philosophie der Orientierung, Berlin, Walter de Gruyter.

Stegmaier, W., 2016, Orientierung im Nihilismus - Luhmann meets Nietzsche, Berlin/Boston, Walter de Gruyter. 
Stolze, R., 1982, Grundlegung der Textübersetzung, Heidelberg, Groos.

Weber, M., 1976, Wirtschaft und Gesellschaft. Grundriss der verstehenden Soziologie., Tübingen, Mohr.

Wittgenstein, L., 2015a, Philosophische Untersuchungen, Frankfurt am Main, Suhrkamp Verlag.

Wittgenstein, L., 2015b, Über Gewissheit, Berlin, Suhrkamp Verlag.

Wolff, L., 2011, Legal Translation. In: Malmkjaer, K. \& Windle, K. (eds.) The Oxford Handbook of Translation Studies. Oxford: Oxford University Press.

Xu, Y. \& Chen, Z., 2006, Relevance judgment: What do information users consider beyond topicality? Journal of the American Society for Information Science and Technology, 57, 961-973.

\section{The concept of relevance and its application in legal translation \\ Summary}

The methodological starting point of this contribution is the concept of equivalence and its criticism in translation theory. The theory of Alfred Schütz and his typology of relevance is underpinning our approach. Subsequently, the intertwining of relevance and orientation will be discussed. In the final part of this paper, we will analyse how relevance is involved in legal translation by providing practical examples of translation from German into French and from French into German.

Keywords: equivalence, relevance, constitution of meaning, orientation, legal translation, interpretation, topic, motivation, creativity 